\title{
Currents and Emerging Technologies for Diabetes Care
}

\author{
Emmanuel Andrès ${ }^{1,2 *}$, Nathalie Jeandidier ${ }^{3}$, Noel Lorenzo Villalba ${ }^{1}$, Laurent Meyer ${ }^{3}$, Abrar Ahmad \\ Zulfiqar $^{1,2}$, Samy Talha ${ }^{2,4}$, Mohamed Hajjam ${ }^{5}$ and Amir Hajjam El Hassani ${ }^{6}$ \\ ${ }^{1}$ Department of Internal Medicine, Diabetes and Metabolic Diseases of Clinique Médicale B, Strasbourg University Hospitals, France
}

${ }^{2}$ Research team EA 3072 “Mitochondria, Oxidative stress and Muscle protection”, Faculty of Medicine of Strasbourg, University of Strasbourg (Unistra), France

${ }^{3}$ Department of Endocrinology and Diabetology of Clinique Médicale B, Strasbourg University Hospitals, France

${ }^{4}$ Department of Physiology and Functional Explorations, Strasbourg University Hospitals, France

${ }^{5}$ Predimed Technology, France

${ }^{6}$ Research team EA 4662 "Nanomedicine, Imaging, Therapeutics”, University of Technology of Belfort-Montbéliard (UTBM), France

*Corresponding author: Emmanuel Andrès, Department of Internal Medicine, Diabetes and Metabolic Diseases of Clinique

Médicale B, Strasbourg University Hospitals, France

\section{ARTICLE INFO}

Received: 㗀 January 23, 2020

Published: 幽 January 30, 2020

Citation: Emmanuel Andrès, Nathalie Jeandidier, Noel Lorenzo Villalba, Laurent Meyer, Abrar Ahmad Zulfiqar, et al. Currents and Emerging Technologies for Diabetes Care. Biomed J Sci \& Tech Res 25(2)-2020. BJSTR. MS.ID.004161.

Keywords: Diabetes Mellitus; Glucose Sensors; Connected Insulin Pens; Intelligent Insulin Pumps; Artificial Pancreas; Telemedicine; Artificial Intelligence; Big Data

\begin{abstract}
In recent years, several technological innovations have or should in the near future become part of the daily lives of diabetic patients as non-invasive glucose sensors, connected insulin pens, intelligent insulin pumps, artificial pancreas, telemedicine, and artificial intelligence. A review of the literature dedicated to these technologies supports the efficacy of these latter in diabetic patients. Mainly, these technologies have shown a beneficial effect on diabetes management with an improvement of: blood glucose control, with a significant reduction in HbA1c; patient ownership of the disease; patient adherence to therapeutic and hygiene-dietary measures; the management of comorbidities (hypertension, weight, dyslipidemia); and at least, good patient receptivity and accountability. Especially, the emergence of these technologies in the daily lives of diabetic patients has led to an improvement of the quality of life for patients. To date, the magnitude of its effects remains debatable, especially with the variation in patients' characteristics, samples selection and approach for treatment of control groups.
\end{abstract}

Abbreviations: ICT: Information and Communication Technologies; MARD: Mean Absolute Relative Difference; AI: Artificial Intelligence; GLP1: Glucagon-Like Peptide-1; ANN: Artificial Neural Networks

\section{Introduction}

Worldwide the number of patients with diabetes mellitus is increasing. In industrialized countries, there are estimations that diabetes is one of the leading causes of death. Today, patient with diabetes spend time each day carefully tracking blood glucose levels, food intake and physical exercise to calculate when and how much insulin should be injected into their bodies. Living with diabetes requires constant vigilance and a strong sense of self-determination and efficacy. In this context, diabetes, as many chronic diseases, benefits from both the contributions of molecular biology and innovative therapies (e.g., new insulins, immunotherapy, stem cell therapy, intestinal microbiote transplantation), and from major advances in technologies (e.g., sensors, infusion systems, connected objects) and in artificial intelligence (e.g., Big Data analysis) [1]. Combined with the Information And Communication Technologies (ICT) and the social and educational sciences, these technological advances will revolutionize the care of diabetic patients in the future [2]. This short narrative review focuses on new and current technologies, used in the field of diabetes mellitus. 


\section{Current Management of the Diabetic Patient}

To date, the management of the diabetic patient is based on a balance of his diabetes (documented by the level of hemoglobin A1c [HbA1c]) with regard to his clinical phenotype, with personalized blood glucose targets [1-3]. Intensive glucose control has been shown to delay or prevent the development of micro- and macrovascular complications related to diabetes [1]. In this context, optimal management of the diabetic patient is based on patient ownership of the disease, therapeutic education, compliance with hygienedietary measures, therapeutic compliance and physical activity $[3,4]$. The last two decades have seen major advances in technology, which has manifested in more accurate glucose monitoring systems and insulin delivery devices ('insulin pump'). Increased understanding of the pathophysiological deficits underlying type 2 diabetes has led to the development of targeted therapeutic approaches such as on the small intestine (glucagon-like peptide-1 receptor analogues and dipeptidyl-peptidase IV inhibitors) and kidneys (sodium-glucose cotransporter-2 inhibitors).

For type 1 diabetic patient, intensive insulin therapy is the reference treatment ("gold standard") [1-3]. In this setting, large multicentre randomized trials have confirmed the effectiveness of intensive glycemic control on microvascular outcomes, but macrovascular outcomes and cardiovascular safety remain controversial with several glucose-lowering agents. Improvements in insulin formulations over the decades, including rapid-acting and long-acting insulin analogues that more closely mimic physiological insulin secretion, have increased the flexibility and efficacy of type 1 diabetes management. Based on studies that have demonstrated the benefits on $\mathrm{HbA1c}$, the frequency of acute hypoglycemic and hyperglycemic episodes, the external rapid analogue infusion pump associated with the Flash Glucose Monitoring ${ }^{\mathrm{TM}}$ system (Abbott Laboratory) (Figure 1) is currently the reference management for patients undergoing intensive insulin therapy [4].

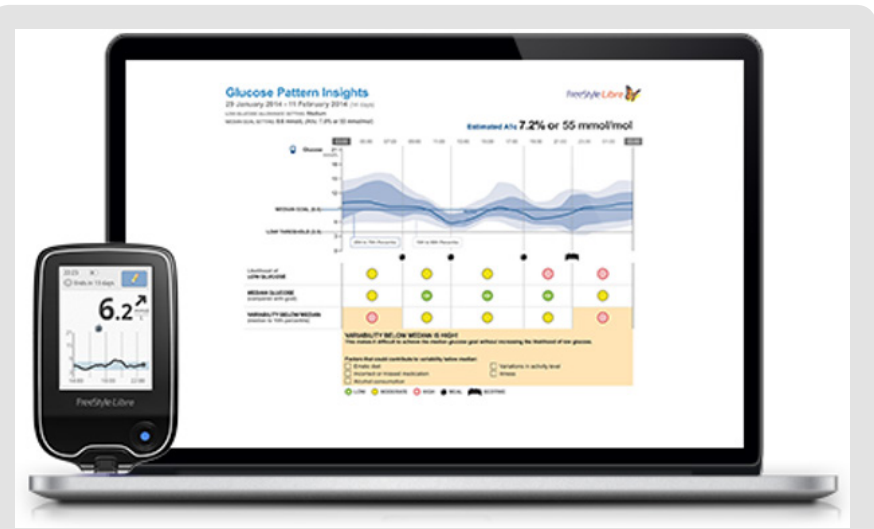

Figure 1: Flash Glucose Monitoring ${ }^{\mathrm{TM}}$ system from Abbott Laboratory.

For the type 2 diabetic patient, it is imperative, in addition to the balance of diabetes (e.g., using metformin, GLP1-agonist drugs or DPP-IV inhibitors recently launched on the market) and the prevention of its complications, to properly manage comorbidities as, overweight, dyslipidemia, arterial hypertension, smoking ant sedentary lifestyle [3].

\section{Non-Invasive Sensors For Glucose Self-Monitoring}

For the diabetic patient, self-monitoring with a capillary blood glucose meter has long been the only way to understand his or her blood glucose control [5]. This self-monitoring gives a more or less truncated reflection of glycemic control (subject to interpretation) and above all allows the patient to adapt his insulin administration. In this setting, Holter glycaemia, followed by realtime continuous glucose measurement in the 2000s, revolutionized our vision of glycemic control [5]. In recent years, non-invasive connected sensors measuring interstitial glucose continuously have become more accurate, gradually freeing themselves from calibration constraints (e.g., Freestyle Libre ${ }^{\mathrm{TM}}$, Abbott Laboratory), or from drug interference (e.g., paracetamol), operating for longer and longer (15 days to 6 months), and becoming more discreet by placing themselves under the skin (Eversense ${ }^{\mathrm{TM}}$, Senseonics/Roche Diabetes Care) $[5,6]$. In the near future, Novo Nordisk's connected insulin pens will integrate with the Abbott Freestyle Libre ${ }^{\mathrm{TM}}$ system, allowing Freestyle Libre $^{\mathrm{TM}}$ users to see data about their insulin alongside their glucose readings. The improvement in their accuracy (meaning Mean Absolute Relative Difference [MARD], from 16-20\% to $10-14 \%$ ) allows direct adaptation of insulin without concomitant control of capillary blood glucose levels [6]. Clinical studies have validated this method, which replaces the classic capillary selfmonitoring of blood glucose in the management of patients treated with intensive insulin therapy.

Controlled clinical studies have shown the efficacy of these devices on the improvement of HbA1c, associated with a decrease in the time spent in hypoglycemia, in type 1 diabetes under external pump, but also under multi-injection (DexCom STSTM System, Dexcom, Inc.) [6]. In addition, their efficacy has also been confirmed in type 2 diabetes, in pregnant women and in children [7]. The connection of the sensors and the possible sharing of data (Dexcom G5TM Mobile, Dexcom, Inc.), allow a joint analysis of these data by the patient, the parents of a child, the doctor or the nurse, thus avoiding, thanks to rapid adaptation of the treatment, deterioration in glycemic control. Interstitial glucose data, glycemic variability, time spent in the target defined for a patient, complete the old "hard" criteria of HbA1c and frequency of hypoglycemia. Thus new guidelines, which will be refined based on clinical studies, may propose in the near future a new definition of glycemic control assessment: "time spent in the target of $0.70-1.80 \mathrm{~g} / \mathrm{L}$ greater than $60 \%$ and time spent in hypoglycemia of less than $10 \%$ " [6]. These criteria perfectly complete $\mathrm{HbA1c}$, a reflection of the glycation of the body's proteins, whose place remains to be redefined. In some industrialized countries (e.g., in France), the reimbursement by health insurance companies of these devices (e.g., FreeStyle ${ }^{\mathrm{TM}}$ Libre, Abbott laboratory), and the soon-to-be-announced reimbursement 
of sensors coupled to external pumps for highly unstable type 1 diabetic patients, opens the way to another modality of the concept of glycemic control assessment [5].

\section{Connected Insuline Pens}

Novo Nordisk recently announced its plans for a connected ("smart") insulin pen, which will automatically record how much insulin was injected. For those on multiple daily injections, this means no logs, no forgetting doses or accidental insulin stacking, and access to the same computer-generated reports that help recognize patterns and optimize therapy as pump users. The new connected pens (NovoPen 6TM and the NovoPen Echo Plus ${ }^{\mathrm{TM}}$ ) are reusable, already approved in Europe (CE marked), and include a tiny screen that displays the last dose. They have piloted with great success in approximately 700 Swedish users with diabetes. A 2019 US launch may be possible, depend on how things go with the FDA. Novo Nordisk's connected insulin pens will integrate with the Abbott Freestyle Libre ${ }^{\mathrm{TM}}$ system, allowing Freestyle Libre ${ }^{\mathrm{TM}}$ users to see data about their insulin alongside their glucose readings (http://www.diabetesincontrol.com/new-smart-pens-hoped-tochange-the-way-we-treat-diabetes/). Lilly has also joined the race to offer tech-enabled, smarter methods of insulin delivery to people with diabetes. Lilly plans to launch two systems: an Automated Insulin Delivery (AID) with Lilly's own custom disk-shaped pump, CGM, and a hybrid closed loop control algorithm; and a smart insulin pens with a dosing decision ("titration") support app. Lilly has been developing both products for two years, and the first trials are expected to begin next month. Dexcom's CGM will be used in both, per an agreement announced in tandem with this news. During a time of fast-paced innovation and competition in the world of diabetes, all three major insulin companies - Lilly, Novo Nordisk, and Sanofi - are investing in digital health and connected delivery devices, though this represents the largest commitment yet. Lilly will bring all the components together for smarter insulin delivery (both pump and injection), submit them to the FDA, and commercialize both systems. Bigfoot Biomedical is currently the only other company pursuing both injection- and pump-based automated delivery of insulin.

\section{Intelligente Insulin Pumps}

For type 1 and numerous type 2 diabetic patients (e.g., type 2 diabetic patients with cardiovascular complications), insulin therapy is the necessary treatment. In this setting, fast or slow insulin analogues are usually administered subcutaneously, with one or more injections per day (e.g., multiple injections in intensive therapy) [3,4]. Recent years, progress has been made with the development of ultra-fast analogues (aspart Fiasp ${ }^{\mathrm{TM}}$, Novo Nordisk Laboratory, recently launched on the French market), which allow the maximum peak action to be advanced and reduce the duration of action, and therefore the quantity of insulin "on board", by about 10 minutes [4]. They will limit the latency between flow rate changes and insulin levels in the blood, improving system performance. Nevertheless, the limitations of subcutaneous administration remain related to the still too long insulin kinetics, the reproducibility of imperfect absorption, and the absence of a first hepatic passage that is physiological. In this context, studies have been carried out with the intraperitoneal route of administration. Compared to the subcutaneous route, this latter improves the $\mathrm{HbA1c}$ and is associated with a decrease in the frequency of severe hypoglycemia [8]. The outer surface of the peritoneum appears to be a promising site, and some bio-artificial pancreases already use this route (e.g., BAir ${ }^{\mathrm{TM}}$, Beta-02 Technologies and MailPan ${ }^{\mathrm{TM}}$ [for MAcrocroencapsulation of PANcreatic ILôts], Defymed Company), with kinetic and metabolic results comparable to those of the intraperitoneal route. An access port device at this site allows for optimized insulin delivery either by an external pump or by injections. On this model, the device ExOlin ${ }^{\mathrm{TM}}$ (Defymed Company) is under development. The connection of the Enlite ${ }^{\mathrm{TM}}$ sensor to the MiniMed $\mathrm{Veo}^{\mathrm{TM}}$ and 640GTM pumps (Medtronic Company) allows the automatic stopping of insulin infusion when a low interstitial glucose concentration is detected or predicted, dramatically reducing the occurrence of severe hypoglycemia (Figure 2) [4]. The recent reimbursement by the health insurance of this system in certain poorly balanced type 1 diabetic patients, subject to severe hypoglycemia under insulin therapy by pump and adapted selfmonitoring, allows for management within the framework of the care of this precursor of the "artificial pancreas".

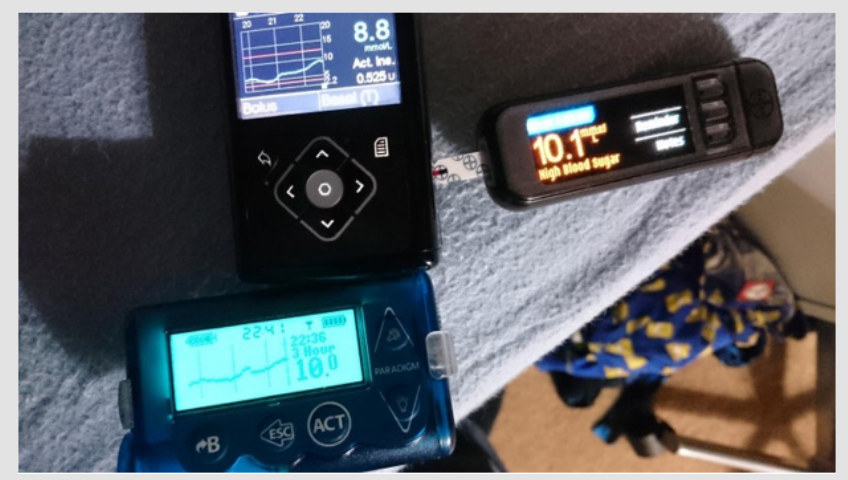

Figure 2: Enlite ${ }^{\mathrm{TM}}$ sensor - MiniMed $\mathrm{Veo}^{\mathrm{TM}}$ - 640GTM pumps from Medtronic Company.

In this context, several "bolus calculators" have been developed, especially for the insulin pumps, offering a bolus dose by coupling the current blood glucose level and a predetermined insulin/ glucose ratio [2]. Nowadays, these systems have been replaced by new intelligent systems based on algorithms (Artificial Intelligence [AI]) [2-7]. These latter make it possible to propose a real adaptation of prandial and basal doses by integrating several parameters (glycaemia, insulin sensitivity, etc.) specific to the patient phenotype (personalized medicine). Self-learning, they are specifically adapted to the patient's history of glycemic variations. They have shown their effectiveness on HbA1c, without increasing hypoglycemia, especially when coupled with nursing "coaching" (Diabeo $^{\mathrm{TM}}$, Sanofi Laboratory) [9]. This system is currently approved within the framework of telemedicine [9]. Coupled with 
an external 670GTM pump (Medtronic Company), other algorithms already allow automatic adaptation of basal rates, with the patient managing only bolus doses [7].

\section{Artificial Pancreas for Glycemic Management}

The rise of all these technologies that we have just seen has led to the recent appearance of the "artificial pancreas", the "diabetic patient's dream" [10]. Since the demonstration, in 2015, of its efficacy in ambulatory care, the results of 24 studies on 585 patients, compiled in a recent meta-analysis, have confirmed a significant improvement in the time spent in the target, the reduction of $\mathrm{HbA1c}$ and mean blood glucose, without an increase in hypoglycemia $[10,11]$. To date, the artificial pancreas is based on a closed-loop insulin delivery system, integrating AI. Most of these devices are mono-hormonal (insulin) and semi-automatic, with the patient manually reporting food intake and physical activity. Many of these devices are expected to be quickly brought to market (e.g., Diabeloop ${ }^{\mathrm{TM}}$ from Medtech Company) [12]. The limitations of single-hormonal subcutaneous devices are related to sensor latency, kinetics of interstitial glucose changes, and reproducibility of peripheral administration of subcutaneous insulin. In this setting, the bi-hormonal approach (insulin-glucagon), poses technical problems, as the stability of glucagon and the necessity of double reserves, but seems interesting to avoid hypoglycemia, especially during physical exercise $[10,11]$. The addition of amylin or Glucagon-Like Peptide-1 (GLP1) receptor analogue improves post-meal blood glucose levels by decreasing glucagon secretion; future years should make it possible to clarify the place of these molecules in the artificial pancreas.

Another approach would be to operate other sites that combine sensors and insulin delivery. A study combining a subcutaneous sensor and intraperitoneal insulin infusion showed better regulation of post-meal periods [13]. Intraperitoneal insulin, which is more physiological, could improve problems related to meals and physical activity. Projects to miniaturize the implantable system and reduce its cost are all assets for make it an attractive alternative. Improving the skills and the capacities of algorithms, by using the databases set up (big data analysis), optimizing their selflearning capacity, their patient-specific adaptation capacity, and supplementing their information with multiple sensors collecting parameters other than blood glucose levels, could allow early detection of food intake, physical activity, stress, and adaptation of the system to specific situations (children, pregnancies, highly unstable diabetes) [14]. The connection of the system to a telemedicine and coaching platform is an evolution that is already underway in the system Diabeloop ${ }^{\mathrm{TM}}$.

\section{Telehealth for Diabetic Patients}

A 2009 study conducted by Julie Polisena and her team at the Canadian Agency for Drugs and Technologies in Health found storing or sharing self-monitored blood glucose using home telehealth tools such as PDAs or fax machines, supported with physician feedback, showed improved glycemic levels and reduced hospitalizations. In this setting, In contrast, a systematic review of the use of cell phones in health promotion strategies found that of the ten studies that looked at cell phones and HbA1c, nine reported significant improvements in the blood glucose control. In addition to improved diabetes-related health outcomes, knowledge, self-efficacy and better adherence to protocol scores were increased in subjects who practiced self-management behaviors. Technology now has evolved beyond telehealth. Smart technology exists as wearables, implants, and mobile applications to track glucose levels, share data, access relevant information, communicate with both healthcare providers and others with diabetes, and, ultimately, guide you in making better decisions. There is an abundance of Smart Apps available today, with a variety of features such as monitoring food intake, carbohydrate intake, tracking physical activity, scanning the barcode of a food product and retrieving its nutritional information as well as offering suggestions for healthier options, healthy recipes, getting signed to create a community database where patients can share their stories etc. Some examples include apps such as Diabetik ${ }^{\mathrm{TM}}$, Fooducate ${ }^{\mathrm{TM}}$ Figwee $^{\mathrm{TM}}$ and MyFitnessPal ${ }^{\mathrm{TM}}$, etc. To our knowledge, more than 350,000 applications are currently available for the general public, without medical CE marking. In addition, several blood glucose monitors can be connected to an app that can be downloaded on a device and track blood glucose numbers as well as any adjustments that need to be made with medications. Some companies such as Glooko have developed Apps that can sync data collected from patient's glucometers and fitness watches to downloadable software that can enable physicians with real-time tracking of patient data.

\section{Telemedecine for Diabetic Patients}

Since the early 1990s to the end of the 2010's, numerous telemedicine projects and studies have been developed in the field of diabetes, especially developed for patient monitoring [2]. Practically all of them have investigated telemonitoring in specific diabetic patients (children and young people, elderly patients, patients with intensified therapy, patients under insulin pump therapy and patients with complicated or complex diabetes). The results of these telemedicine projects (including type 1 and type 2 diabetic patients, involving the upload and direct transmission of blood-glucose data by diabetic patients to providers via cellular telephone, telephone land line, or a Web-based program) differed from study to study, with fairly inconclusive results as to their potential clinical benefits in terms of balancing diabetes (Table 1) [15]. This is also the case in terms of: the management of associatedmetabolic problems and comorbidities; re-hospitalization; and decreased morbidity or mortality, particularly regarding the statistical significance of the results. Over the last ten years, "new" generation telemedicine projects and studies have been developed in the setting of diabetes management [2]. These projects and studies have for main objectives to evaluate the use of technology to implement medical and cost-effective health care management 
on a large scale for diabetes management. Compared to the first projects, most of these new generation projects incorporate: selfadministered medical questionnaires or forms on: symptoms, signs of diabetes decompensation; tools for medical education, particularly disease self-appropriation, food hygiene, and physical activity; tools for patient motivation; tools for therapeutic and hygiene observance; tool to remote comorbidities (e.g., arterial hypertension, obesity, dyslipidemia); tools for interaction between the patient and healthcare professionals like telephone support centers, tablets, and Web-sites [2].

Table 1: Results of the telemonitoring studies conducted in the field of diabetes during the period from 2010 to 2015 [2,15].

\begin{tabular}{|c|c|}
\hline Name of the study & Results \\
\hline $\begin{array}{l}\text { The Utah Remote Monitoring } \\
\text { Project } \\
(n=109)\end{array}$ & $\begin{array}{l}\text { Principal criteria: } \\
\text { - Mean HbA1c had decreased from } 9.73 \% \text { at baseline to } 7.81 \% \text { at the end of the program }(\mathrm{p}<0.0001) \\
\text { - Systolic blood pressure (BP) had decreased from } 130.7 \mathrm{mmHg} \text { at baseline to } 122.9 \mathrm{mmHg} \text { at the end } \\
\qquad(\mathrm{p}=0.0001) \\
\text { Secondary criteria: } \\
\text { - Low- density lipoprotein content had decreased from } 103.9 \mathrm{mg} / \mathrm{dL} \text { at baseline to } 93.7 \mathrm{mg} / \mathrm{dL} \text { at the end } \\
\qquad(\mathrm{p}=0.0263) \\
\text { - Knowledge of diabetes and arterial hypertension have increased significantly (p <0.001 for both). } \\
\text { - Patient engagement and medication adherence also have improved, but not significantly }\end{array}$ \\
\hline $\begin{array}{l}\text { Randomized Trial on Home } \\
\text { Telemonitoring for the } \\
\text { Management of Metabolic } \\
\text { and Cardiovascular Risk in } \\
\text { Patients with type } 2 \text { Diabetes } \\
\qquad(n=302)\end{array}$ & $\begin{array}{l}\text { Principal criteria: } \\
\text { - Mean HbA1c difference of } 0.33 \pm 0.1(\mathrm{p}=0.001) \text { have been observed between the telemonitoring compared } \\
\text { and the control group. The proportion of patients reaching the target of HbA1c }(\mathrm{HbA} 1 \mathrm{c}<7.0 \%) \text { had } \\
\text { been higher in the telemonitoring group than in the control group after } 6 \text { months: } 33.0 \% \text { vs. } 18.7 \% \\
(\mathrm{p}=0.009) \text { and } 12 \text { months: } 28.1 \% \text { vs. } 18.5 \%(\mathrm{p}=0.07) . \\
\text { - No difference had been registered for body weight, BP, and lipid profile } \\
\text { Secondary criteria: } \\
\text { - For quality of life (evaluated with the } 36 \text {-item Short Form health survey), significant differences in favor of } \\
\text { the telemonitoring group, as for physical functioning (p=0.01) and mental health (p=0.005). } \\
\text { - On an economic level, a lower number of specialist visits was reported in the telemedicine group: inci- } \\
\text { dence rate ratio of } 0.72 \text { ( } 95 \% \text { confidence interval, } 0.51-1.01 \text {; } \mathrm{p}=0.06) .\end{array}$ \\
\hline $\begin{array}{l}\text { Study assessed the utility } \\
\text { and cost-effectiveness of an } \\
\text { automated Diabetes Remote } \\
\text { Monitoring and Management } \\
\text { System (DMRS) } \\
(n=98)\end{array}$ & $\begin{array}{l}\text { Principal criteria: } \\
\text { - No significant difference for mean HbA1c between the DRMS and control groups at } 3 \text { months: } 7.60 \% \text { vs. } \\
8.10 \% \text { and at } 6 \text { months: } 8.10 \% \text { vs. } 7.90 \%(\mathrm{p}=\mathrm{ns}) \\
\text { Secondary criteria: } \\
\text { - Changes from baseline to } 6 \text { months have been not statistically significant for self-reported medication } \\
\text { adherence } \\
\text { - Changes of diabetes-specific quality of life have been not significant registered, except for the Daily Quality } \\
\text { of Life-Social/Vocational Concerns subscale score (p=0.04) }\end{array}$ \\
\hline $\begin{array}{l}\text { Telescot Diabetes Pragmatic } \\
\text { Multicenter Randomized } \\
\text { Controlled Trial } \\
(n=321)\end{array}$ & 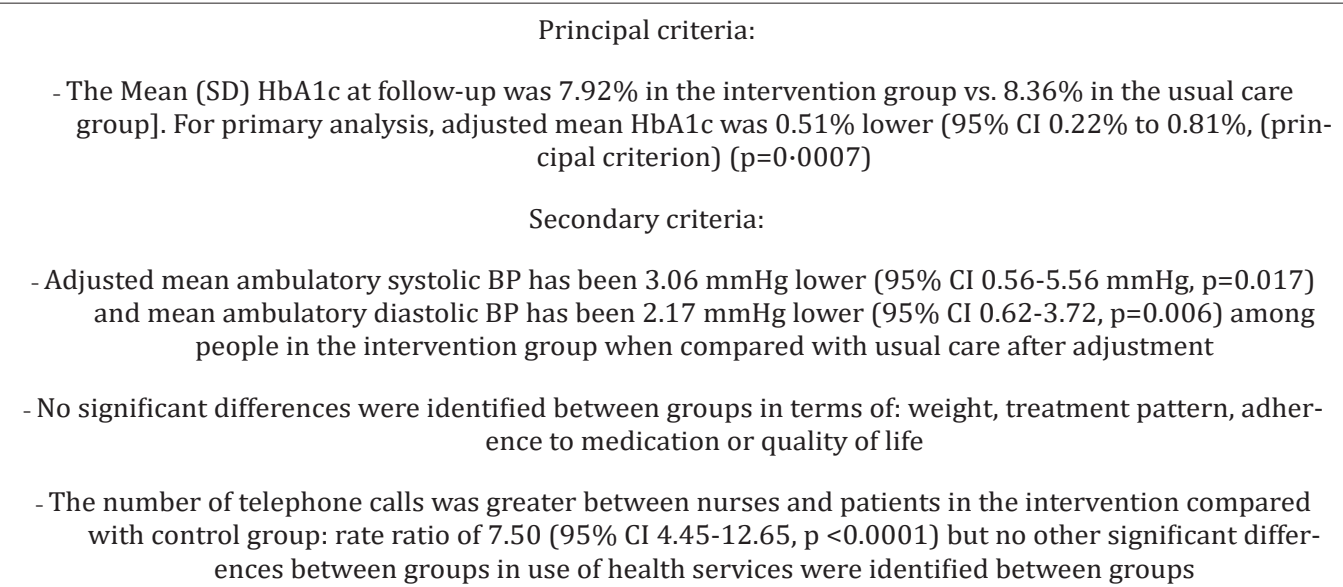 \\
\hline
\end{tabular}


The analysis of these different projects and studies shows that remote monitoring (telemonitoring) showed: improvements in control of blood glucose level, significant reduction in $\mathrm{HbA1c}$; better appropriation of the disease by patients; greater adherence to therapeutic and hygiene-dietary measures; positive impact on comorbidities (arterial hypertension, weight, dyslipidemia); better patient's quality of life; and at least, good receptiveness by patients and patient empowerment [2]. Moreover, a cost-effectiveness analysis found a potential of medical economy. However, to date, the magnitude of its effects remains debatable, especially with the variation in patients' characteristics (e.g., background, ability for self-management, medical condition), samples selection and approach for treatment of control groups. Over the last 5 years, new-generation telemedicine projects and studies have emerged in the setting of type 1 and type 2 diabetes [2-9,16,17]. They support transmission and remote interpretation of patients' data for follow-up and preventive interventions. These new generation telemedicine projects are often known as "telemedicine 2.0" projects, given that they all utilize new Information and Communication Technologies (ICT) and the Web (tools for the "e-Health 2.0") [18]. These projects rely on the standard connected tools for monitoring diabetes, such as glucose meters, BP, heart rate monitors, weighing scales, and pulse oximeters, which relay the collected information via Bluetooth, 3G or 4G [2-19]. They include continuous glycemic monitoring solution and often a video-call.

\section{Artificial Intelligence for Diabetes Management}

In recent years, several informatics solutions or tools have been developed and used to optimize the management of chronic disease, such as: Artificial Neural Networks (ANN) algorithms, data mining software, ontology [2-20]. These solutions or tools are called Artificial Intelligence (AI) and are the are the support of "telemedicine 3.0". For this later, three clinical datasets are of particular interest:

1. patients' phenotype;

2. patients' electronic medical records containing physicians' notes, laboratory test results, as well as other information on diseases, treatments, and epidemiology that may be of interest for association studies and predictive modeling on prognosis and drug responses; and

3. literature knowledge including rules on diabetes management [20].

In the setting of diabetes, two of the aforementioned telemedicine projects use AI in order to be able: firstly, to adjust the blood glucose level to the patient's activity (software Diabeo ${ }^{\mathrm{TM}}$, Sanofi Laboratory) [9]; and secondly, to predict patient risks of diabetes decompensation. In this later situation, the cloud-based software aggregates, cleans, and analyzes patient data to allow for identifying patterns that may indicate potential risks and provide predictive insights on healthcare outcomes, as the software MyPredi $^{\mathrm{TM}}$ (Predimed Technology Company). In the TELESAGE study, type 1 diabetic patients were randomized to usual quarterly follow-up (G1), home use of a smartphone recommending insulin doses (Diabeo ${ }^{\mathrm{TM}}$ software) with quarterly visits (G2), or use of the smartphone with short teleconsultations every 2 weeks but no visit until point end (G3) [9-17]. At six-month, the mean HbA1c level: $8.41 \pm 1.04 \%$ in G3 vs. $8.63 \pm 1.07 \%$ in G2 vs. $9.10 \pm 1.16 \%$ in G1 ( $\mathrm{p}=0.0019$ for G1-G3 comparison). The Diabeo ${ }^{\mathrm{TM}}$ system gave a $0.91 \%(0.60-1.21)$ improvement in HbA1c over controls and a $0.67 \%(0.35-0.99)$ reduction when used without teleconsultation. There was no difference in the frequency of hypoglycemic episodes or in medical time spent for hospital or telephone consultations. However, patients in G1 and G2 spent nearly $5 \mathrm{~h}$ more than G3 patients attending hospital visits.

The DIABETe telemonitoring project, has been developed and designed to optimize home monitoring of diabetic patients by detecting, via a telemonitoring 2.0 platform, situations with a risk of decompensation of diabetes and its complications (e.g., myocardial infarction or chronic heart failure), the latter ultimately leading to hospitalization (https://www.predimed-technology.fr/solutions/ plateforme-intelligente-my-predi/). The AI of the DIABETe platform (MyPredi ${ }^{\mathrm{TM}}$, tool of telemedicine 3.0) automatically generates indicators of "health status" deterioration, i.e., "warning alerts" for any chronic disease worsening, particularly diabetes, its macrovascular complications and cardiovascular comorbidities (e.g., arterial hypertension, chronic heart failure). For the patient, these situations may lead to hospitalization if not treated appropriately. To our knowledge, this is one of the first projects that use AI in addition to ICT (telemedicine 3.0). The platform comprises connected nonintrusive medical sensors, a touchscreen tablet connected by $\mathrm{Wi}-\mathrm{Fi}$, and a router or $3 \mathrm{G} / 4 \mathrm{G}$, rendering it possible to interact with the patient and provide education on treatment, diet, and lifestyle (Figure 3).The telemonitoring platform used in DIABETe was first experimented in a monocentric study conducted in the Strasbourg University Hospital, carried out as part of the E-Care project, primarily focused on the problem of chronic heart failure [21]. Between February 2014 and April 2015, 175 patients (mean age of 72 years) were included into the E-care project, $30 \%$ of these patients suffered from type 2 diabetes. During this period, the telemonitoring platform was used on a daily basis by patients and healthcare professionals, according to a defined protocol of use specific to each patient. During the study, 1,500 measurements were taken, generating 700 alerts in 68 patients. 107 subjects (61.1\%) had no alerts upon follow-up. Analysis of the warning alerts in the 68 other patients showed that MyPredi ${ }^{\mathrm{TM}}$ detected any worsening of the "patient's health", with a sensitivity, specificity, as well as positive and negative predictive values of: $100 \%, 30 \%, 89 \%$ and $100 \%$, respectively. In this experimentation, both the healthcare professionals and patients, even the frailest, used the E-care system without difficulty until the end of the study. In this setting of IA, all new connected sensors collect data on a daily basis, which are stored and analyzed by Big Data algorithms such as Machine Learning, which will make it possible to predict risk situations, 
investigate their causes and highlight new alternatives for care procedures [20]. The aim is to draw the caregiver's attention to the right patient at the right time and thus avoid an emergency consultation or even hospitalization.

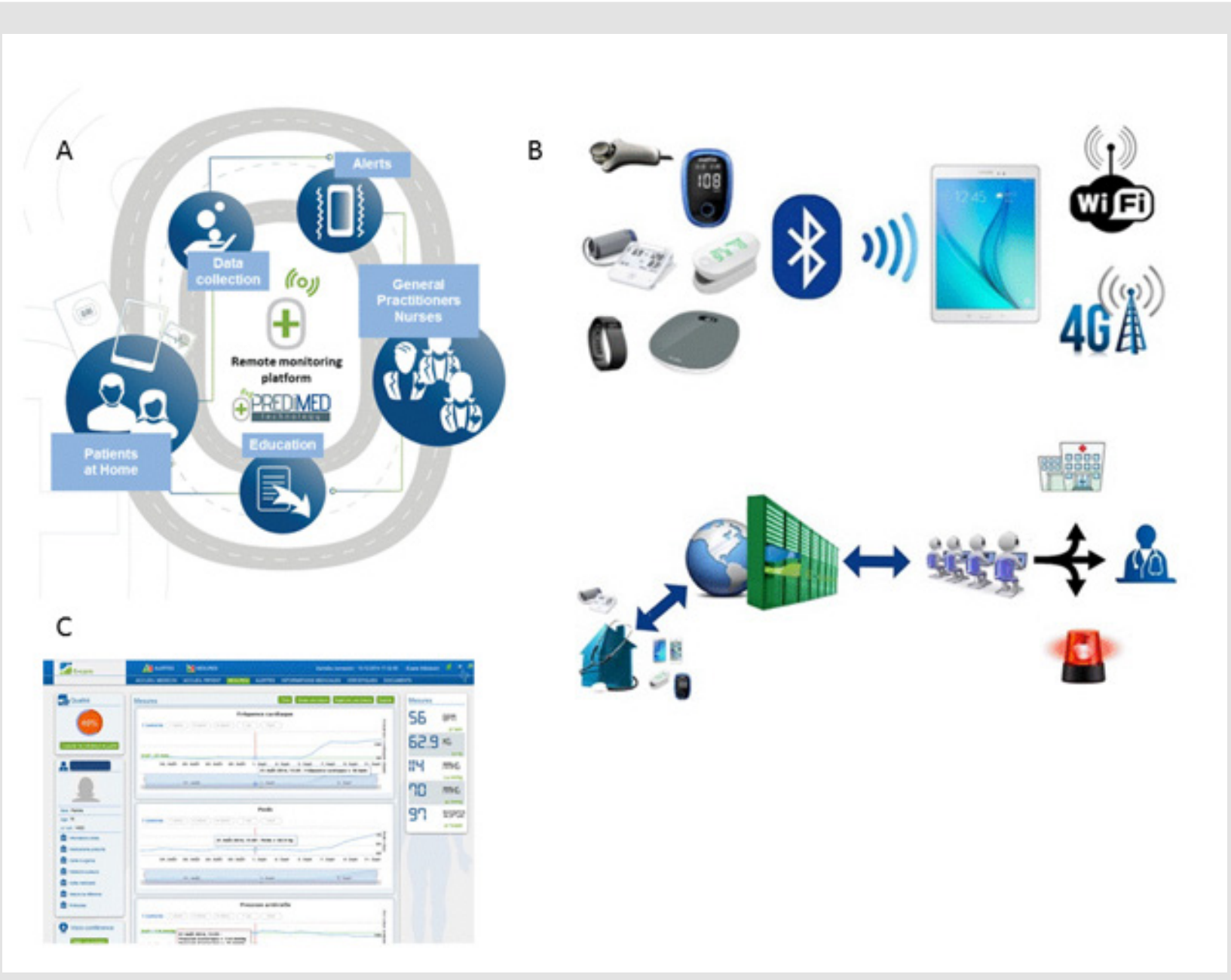

Figure 3: Telemedicine project: DIABETe.

a) A: DIABETe is based on a smart system comprising an inference engine and a medical ontology for personalized synchronous or asynchronous analysis of data specific to each patient and, if necessary, the sending of an artificial intelligencegenerated alert (MyPredi ${ }^{\mathrm{TM}}$, main tool of telemedicine 3.0).

b) B: The platform comprises connected nonintrusive medical sensors, a touchscreen tablet connected by Wi-Fi, and a router or $3 \mathrm{G} / 4 \mathrm{G}$, rendering it possible to interact with the patient and provide education on treatment, diet, and lifestyle.

c) C: The system involves a server that hosts the patient's data and a secure internet portal to which the patient and hospital- and non-hospital-based healthcare professionals can connect.

The current upgraded version of E-care AI had led to the development of MyPredi ${ }^{\mathrm{TM}}$, the main tool (AI) of our current telemedicine 3.0 project, called DIABETe. To date, MyPredi ${ }^{\mathrm{TM}}$ is able to follow several pathologies in the same patient, in particular diabetes, heart failure, hypertension, geriatric risks, etc.

\section{Conclusion}

This short pragmatic narrative review supports the potential interest of numerous technologies as non-invasive glucose sensors, connected insulin pens, intelligent insulin pumps, artificial pancreas, telemedicine, $\mathrm{AI}$, in the field of diabetes mellitus. Mainly, these technologies have shown a beneficial effect on diabetes management with an improvement of: blood glucose control, with a significant reduction in $\mathrm{HbA1c}$; patient ownership of the disease; patient adherence to therapeutic and hygiene-dietary measures; the management of co-morbidities (hypertension, weight, dyslipidemia); and at least, good patient receptivity and accountability. Especially, the emergence of these technologies in the daily lives of diabetic patients has led to an improvement of the quality of life for patients. To date, the magnitude of its effects remains debatable, especially with the variation in patients' characteristics, samples selection and approach for treatment of control groups. Innovative technologies based on AI (machine learning, Big Data) are going to build the future of diabetology ("diabelology 3.0"); fully automated 
artificial pancreas, telemedicine interventions preventing severe glucose degradations and helping with diabetes burden in a day-today basis. Moreover, these technologies will also be a major source to understand mechanisms of disease degradation and psychology and behavior of patients who have to cope with this. This will lead to a new optimized way of patient and disease management. Diabetologists will have to adapt to this new world.

\section{Funding}

Grants from the Agence Régionale De Santé (ARS) du Grand-Est and Fondation d'Avenir.

\section{Competing Interest}

M. Hajjam is the scientific director of Predimed Technology (www.predimed-technology.fr). All other authors have declared that no competing interests exist.

\section{Contributor Ship}

EA and NJ designed the paper and conducted the literature searches. EA, NJ, LM, and MH drafted the results and parts of the discussion. AAZ, ST and AEHH provided critical analysis, revised the whole manuscript, and approved the final version for publication. EA is responsible for all revisions and remains in contact with the rest of the review team regarding status reports.

\section{References}

1. Bartlett ST, Markmann JF, Johnson P, Korsgren O, Hering BJ, et al. (2016) Report from IPITA-TTS Opinion Leaders Meeting on the future of B-cell replacement. Transplantation 100(Suppl 2): S1-S44.

2. Andrès E, Hajjam M, Talha $S$ (2018) Telemedicine in the field of heart failure. State of play and focus on the telemedicine $2.0 \mathrm{E}$-care project. Perspectives in the field of diabetology. Metabolic Diseases Medicine 12: 224-231.

3. Nathan DM (2015) Diabetes: Advances in Diagnosis and Treatment. JAMA 314(10): 1052-1062.

4. Benhamou PY, Lablanche S (2018) Type 1 diabetes: perspectives technologies. MCED 92: 11-16.

5. Rodbard D (2017) Continuous Glucose Monitoring: A review of recent studies demonstrating improved glycemic outcomes. Diabetes Technol Ther 19(Suppl 3): S25-S37.

6. Borot S, Benhamou PY, Atlan C, Bismuth E, Bonnemaison E, et al. (2018) Practical implementation, education and interpretation guidelines for continuous glucose monitoring: A French position statement. Diabetes Metab 44(1): 61-72.

7. Biester T, Kordonouri O, Holder M, Remus K, Kieninger-Baum D, et al. (2017) "Let the algorithm do the work": reduction of hypo- glycemia using sensor-augmented pump therapy with predictive insulin suspension (SmartGuard) in pediatric type 1 diabetes patients. Diabetes Technol Ther 19(3): 173-182.

8. Barrientos R, Baltrusch S, Sigrist S, Legeay G, Belcourt A, et al. (2009) Kinetics of insulin secretion from MIN6 pseudoislets after encapsulation in a prototype device of a bioartificial pancreas. Horm Metab Res 41(1): 5-9.

9. Jeandidier N, Chaillous L, Franc S, Benhamou PY, Schaepelynck P, et al. (2018) DIABEO App Software and Telemedicine Versus Usual FollowUp in the Treatment of Diabetic Patients: Protocol for the TELESAGE Randomized Controlled Trial. JMIR Res Protoc 7(4): e66.

10. Castle JR, DeVries JH, Kovatchev B (2017) Future of automated insulin delivery systems. Diabetes Technol Ther 19(S3): S67-S72.

11. Bertachi A, Ramkissoon CM, Bondia J, Vehí J (2018) Automated blood glucose control in type 1 diabetes: A review of progress and challenges. Endocrinol Diabetes Nutr 65(3): 172-181.

12. Christiansen SC, Fougner AL, Stavdahl Ø, Kölle K, Ellingsen R, et al. (2017) A review of the current challenges associated with the development of an artificial pancreas by a double subcutaneous approach. Diabetes Ther 8(3): 489-506.

13. Renard E, Place J, Cantwell M, Chevassus H, Palerm CC (2010) Closedloop insulin delivery using a subcutaneous glucose sensor and intraperitoneal insulin delivery: feasibility study testing a new model for the artificial pancreas. Diabetes Care 33(1): 121-127.

14. Aleppo G, Webb K (2018) Continuous Glucose Monitoring Integration in Clinical Practice: A Stepped Guide to Data Review and Interpretation. J Diabetes Sci Technol 13(4): 664-673.

15. Andrès E, Talha S, Jeandidier N, Meyer L, Hajjam M, et al. (2018) Telemedicine in Chronic Diseases: The Time of Maturity with Telemedicine 2.0 in the Setting of Chronic Heart Failure and Diabetes Mellitus! Curr Res Diabetes Obes J 6: 1-4.

16. Lindberg I, Torbjørnsen A, Söderberg S, Ribu L (2017) Telemonitoring and Health Counseling for Self-Management Support of Patients With type 2 Diabetes: A Randomized Controlled Trial. JMIR Diabetes 2(1): e10.

17. Charpentier G, Benhamou PY, Dardari D, Clergeot A, Franc S, et al. (2011) The Diabeo software enabling individualized insulin dose adjustments combined with telemedicine support improves HbA1c in poorly controlled type 1 diabetic patients: A 6-month, randomized, openlabel, parallel-group, multicenter trial (TeleDiab 1 Study). Diabetes Care 34(3): 533-539.

18. Van De Belt TH, Engelen L, Berben S, Schoonhoven L (2010) Definition of Health 2.0 and Medicine 2.0: A Systematic Review. J Med Internet Res 12(2): e18.

19. Puricel SG, Ruiz J (2014) Diabetes mellitus and the era of telemedicine. Rev Med Suisse 10(233): 1246-1248.

20. Rjeily CB, Badr G, El Hassani AH, Andrès E (2018) Medical Data Mining for Heart Diseases and the Future of Sequential Mining in Medical Field. Machine Learning Paradigms: Advances in Data Analytics 149: 71-99.

21. Andrès E, TalhaS, Hajjam M, Hajjam J, Ervé S, etal. (2018) Experimentation of 2.0 telemedicine in elderly patients with chronic heart failure: A study prospective in 175 patients. Eur J Intern Med 51: e11-e12. 


\section{ISSN: 2574-1241}

DOI: $10.26717 /$ BJSTR.2020.25.004161

Emmanuel Andrès. Biomed J Sci \& Tech Res

(c) (P) This work is licensed under Creative BY Commons Attribution 4.0 License

Submission Link: https://biomedres.us/submit-manuscript.php

$\begin{array}{ll}\text { BIOMEDICAL } & \text { Assets of Publishing with us } \\ \text { RESEARCHES } & \text { - Global archiving of articles } \\ & \text { - Immediate, unrestricted online access } \\ & \text { - Rigorous Peer Review Process } \\ \end{array}$

